\title{
Insight and Associated Factors among Patients with Schizophrenia in Mental Specialized Hospital, Ethiopia, 2018
}

\author{
Mandaras Tariku, ${ }^{1}$ Demeke Demilew $\mathbb{D}^{\mathrm{D}},{ }^{2}$ Tolesa Fanta, ${ }^{3}$ Meskerem Mekonnen, ${ }^{3}$ \\ and Dessie Abebaw Angaw (D) ${ }^{4}$ \\ ${ }^{1}$ Department of Psychiatry, College of Health and Medical Science, Haramaya University, Harar, Ethiopia \\ ${ }^{2}$ Department of Psychiatry, College of Medicine and Health Science, University of Gondar, Gondar, Ethiopia \\ ${ }^{3}$ Amanuel Specialized Mental Hospital, Addis Ababa, Ethiopia \\ ${ }^{4}$ Department of Epidemiology and Biostatistics, Institute of Public Health, College of Medicine and Health Science, \\ University of Gondar, Gondar, Ethiopia
}

Correspondence should be addressed to Dessie Abebaw Angaw; dessieabebaw96@gmail.com

Received 12 June 2019; Accepted 9 October 2019; Published 17 November 2019

Academic Editor: James Grutsch

Copyright (C) 2019 Mandaras Tariku et al. This is an open access article distributed under the Creative Commons Attribution License, which permits unrestricted use, distribution, and reproduction in any medium, provided the original work is properly cited.

Background. Insight is the degree of the patient's awareness and understanding of their attributions, feelings, behavior and disturbing symptoms. Majority of the patients with schizophrenia have poor insight and insight is an important prognostic indicator in schizophrenia to enhance treatment compliances and reducing the risks of clinical deterioration. The main objective of this study was to assess insight and its associated factors among patients with schizophrenia at mental specialized hospital in Ethiopia. Methods. Institutional based cross-sectional study was conducted from May to June 2018 Mental Specialized Hospital among 455 patients with schizophrenia. Insight was measured by an abridged version of Scale to assess unawareness of mental disorder. Positive and Negative Syndrome Scale, Calgary depressive scale, Oslo social support scale was used to identify factors associated with insight. Simple and multiple linear regression analysis were used to assess associated factors of insight in the participants. Results. The mean score of insight was 13.66 (95\% CI 13.27, 14.04). Age at first onset of illness, duration of treatments, depressive symptoms were inversely associated with mean insight score; whereas unemployed, positive and negative syndrome, previous hospitalization, $>=2$ episodes were positively associated with mean insight score. Conclusion. Nearly half of the study participants were scored above the mean insight score so, the clinicians and psychotherapists should have to work together to improve insight among patients with schizophrenia.

\section{Background}

Insight in mental health is the degree of the patient's awareness and understanding of their attributions, feelings, behavior and disturbing symptoms; self-understanding as well as the potential causes of psychiatric presentation [1].

Insight is viewed as multidimensional variable of the realization of the need for treatments, ability to re-label unusual mental events as pathological and attribute appropriate causes for mental illness. Insight assessment is an important aspect of diagnosis, treatments and prognosis of the patient with mental illness [2-4].

Unawareness of having mental illness among the patients with schizophrenia viewed as an independent phenomenon rather than the secondary manifestation of schizophrenia symptom $[5,6]$.

WHO international pilot study showed that $98 \%$ patients with schizophrenia had a lack of insight and systematic study revealed that between $50 \%$ and $80 \%$ of patients with schizophrenia had characterized by poor insight $[3,7,8]$.

The consequences of lack of insight are failure to recognize the need for treatment; leading to medication nonadherence and impacts on the patients, families, communities and affects the course of illness $[9,10]$.

Insight in the patient with schizophrenia has been associated with the depressive symptoms, positive and negative syndromes of schizophrenia, unemployed, relapse and rehospitalization [11-13]. 
Female and depressive symptoms are associated with good insight whereas symptoms severity, positive and negative syndrome are associated with poor insight $[13,14]$.

Although insight assessment is a crucial aspect of patients with schizophrenia there are few published studies in Africa and no published study in Ethiopia. Therefore, the main aim of this study was assessing insight and its associated factors among patients with schizophrenia at mental specialized hospital in Ethiopia.

\section{Methods}

2.1. Study Setting and Participants. An institutional based cross-sectional study was conducted from May to June 2018 G.C at Amanuel Mental Specialized Hospital located in Addis Ababa. The hospital provides care for patients coming from the entire nation of Ethiopia. Among 3420 patients with schizophrenia who had regular follow up at AMSH; 455 were recruited for the study and those acutely ill and patients with intellectual disability who had schizophrenia were excluded from the study. The samples were selected through systematic random sampling with the interval of 8 .

2.2. Instruments. Structured clinical interview for DSM-4 (SCID) was used to confirm a diagnosis of schizophrenia. Insight was measured through interviews by using an abridged version of scale to assess unawareness of mental disorder (SUMD). It has 9 items self-report measure: awareness of the mental disorder, awareness of achieved effect of medication, awareness of social consequence of mental disorder and awareness of recognition of symptoms rated as 0 (not indicated), 1 (aware), 2 (somewhat aware/unaware ), 3 (severely unaware) and the Cronbach's alpha was 0.89 and the high scores indicates poor insight [15]. Positive syndrome: based on positive syndrome scale of PANSS (7-49) higher scores indicate the presence and severity of positive syndrome, Negative syndrome: based on Negative syndrome scale of PANSS (7-49) higher scores indicates the presence and severity of negative syndrome, Depressive symptoms: patients with schizophrenia scoring $>6$ using Calgary depression scale for schizophrenia, Social support: based on Oslo social support scale scores between (3-8) are poor social support, (9-11) moderate social support and (12-14) strong social support [16-20].

2.3. Data Processing and Analysis. Data were checked for completeness and consistency and entered into Epidata version 3.1 and analyzed by using statistical package software for social sciences (SPSS) version 20. Simple and multiple linear regression analysis were carried out to explain the association between explanatory and outcome variables. The strength of association was measured by $\beta$ coefficient with $95 \%$ confidence interval and the Level of significance was declared at the $p$-value of $<0.05$.

\section{Results}

3.1. General Description of the Study Participants. A total of 445 patients responded to the questionnaire, which yields a
TABLE 1: Description of socio-demographic characteristics of patients with schizophrenia.

\begin{tabular}{|c|c|c|c|}
\hline Variables & Category & Frequency & Percentage \\
\hline \multirow{2}{*}{ Sex } & Female & 170 & 38.2 \\
\hline & Male & 275 & 61.8 \\
\hline \multirow{3}{*}{ Religion } & Orthodox & 211 & 47.4 \\
\hline & Muslim & 179 & 40.2 \\
\hline & Others* & 55 & 12.4 \\
\hline \multirow{4}{*}{$\begin{array}{l}\text { Marital } \\
\text { status }\end{array}$} & Single & 222 & 49.9 \\
\hline & Married & 144 & 32.4 \\
\hline & Separated & 45 & 10.1 \\
\hline & Others $^{* *}$ & 34 & 7.6 \\
\hline \multirow{4}{*}{ Ethnicity } & Amhara & 170 & 38.2 \\
\hline & Oromo & 131 & 29.4 \\
\hline & Gurage & 91 & 20.4 \\
\hline & Others ${ }^{* * *}$ & 53 & 11.9 \\
\hline \multirow{5}{*}{$\begin{array}{l}\text { Educational } \\
\text { status }\end{array}$} & Unable to read and write & 142 & 31.9 \\
\hline & Able to read and write & 204 & 45.8 \\
\hline & Primary school & 43 & 9.7 \\
\hline & High school & 24 & 5.4 \\
\hline & $\begin{array}{c}\text { College graduate and } \\
\text { above }\end{array}$ & 32 & 7.2 \\
\hline \multirow{7}{*}{ Occupation } & Government employed & 88 & 19.8 \\
\hline & Merchant & 47 & 10.6 \\
\hline & Farmer & 47 & 10.6 \\
\hline & Student & 49 & 11 \\
\hline & Day laborer & 29 & 6.5 \\
\hline & House wife & 21 & 4.7 \\
\hline & Unemployed & 164 & 36.9 \\
\hline
\end{tabular}

Others $^{*}$; catholic, protestant others ${ }^{* *}$; widowed, divorced others ${ }^{* * *}$; Tigre, Silte, Woleyta.

response rate of $97.8 \%$. The mean age of the respondents was $36.1 \pm 47.8$ years. Majority of the participants were male and $164(36.9 \%)$ were unemployed (Table 1$)$.

3.2. Clinical Characteristic of the Study Participants. Among the total of 445 participants, 259 (58.2\%) had more than two episodes of schizophrenia and 240 (53.9\%) had previous history of hospitalization. The mean age of the first onset of schizophrenia was $25.7 \pm 5.2$ years and the mean duration of treatment was $6.64 \pm 3.51$ years. The mean of the positive and negative syndromes were $22.67 \pm 6.12$ and $22.36 \pm 3.69$ respectively and $111(24.9 \%)$ had depressive symptoms and majority of the participants were nonadherent to the medication (Table 2).

\subsection{Social Support Characteristics of Study Partici-} pants. Among the total of the study participants, 264 (59.3\%) had poor social support, $74(16.6 \%)$ had moderate social support and 107 (24\%) had good social support.

\subsection{Scale to Assess Unawareness of Mental Disorder} (SUMD). The mean total score of insight was $13.66 \pm 4.21$ $(95 \%$ CI 13.27, 14.04) as measured in a range from 3 to 27 using SUMD an abridged version and (49.2\%) of the participants were scored above the mean total insight score. 
TABLE 2: Description of clinical characteristic of patients with schizophrenia.

\begin{tabular}{lccc}
\hline Variables & Category & Frequency & Percentage (\%) \\
\hline Number of episodes & First episode & 186 & 41.8 \\
& $\geq 2$ episodes & 259 & 58.2 \\
Previous & Yes & 240 & 53.9 \\
hospitalization & No & 205 & 46.1 \\
Depressive & Yes & 111 & 24.9 \\
symptoms & No & 334 & 75.1 \\
Age at first onset of & & 25.70 & \\
illness mean (SD) & & $(5.22)$ & \\
Duration of & & $6.64(3.51)$ & \\
treatment mean (SD) & & & \\
Positive syndrome & & 22.67 & \\
mean (SD) & & $(6.12)$ & \\
Negative syndrome & & 22.36 & \\
mean (SD) & & $(3.69)$ & \\
General & & 27.42 & \\
psychopathology & & $(10.45)$ & \\
mean (SD) & & 72.44 & \\
PANSS total mean & & $(14.00)$ & \\
(SD) & &
\end{tabular}

Based on multivariate analysis $88.1 \%$ of the variation of insight was explained by the variables in the model. The mean insight score of study participants who were unemployed was increased by 1.50 units $(95 \%$ CI $1.09,1.92)$ as compared with those who were government employed and the study participants who had more than two episodes had their mean insight score increased by 0.95 units $(95 \% \mathrm{CI} 0.61,1.29)$ as compared with those who had the first episode (Table 3 ).

\section{Discussion}

The mean total score of insight among patients with schizophrenia was $13.66 \pm 4.21(95 \%$ CI $13.27,14.04)$ and $49.2 \%$ of the participants were scored above the mean score. The result was supported by a cross-sectional study in Peru (13.28 \pm 8.678$)$ [21], Taiwan (13.1 \pm 7.4$)$ [22] and Netherlands (13.6 46.7$)$ [23]. The current finding was higher than a cross-sectional study conducted in Malawi (6.8 \pm 4.1$)$ [24], Ghana (8.32 \pm 6.22$)$ [25] and China $(9.7 \pm 5.6)[26]$ which showed that the total mean insight score of the participants were low. The possible reasons could be sample size difference (60 in Malawi, 49 in Ghana, 139 in china) and measurement difference in assessing insight (Schedule for assessment of insight in both Malawi and Ghana study whereas, Insight and treatment attitude questionnaires in China. The result also not supported by a cross-sectional study in Nigeria $(9.6 \pm 4.7)$ [27] by using SUMD. This discrepancy could be justified as the study participants were low mean scores of psychopathology (the mean positive and negative syndrome was 13.08 and 19.92 respectively), sample size differences (312 samples), excludes new patients and participants' take medication for more than 1 year included in the Nigeria study.

The current study revealed that being unemployed was positively associated with the mean total insight score. The result was in agreement with a comparative study conducted
TABLE 3: Factors associated with insight among patients with schizophrenia at mental specialized hospital, Addis Ababa, Ethiopia June, 2018.

\begin{tabular}{lc}
\hline Variables & $\beta$ coefficient $(95 \% \mathrm{CI})$ \\
\hline Male & $0.15(-1.50,0.44)$ \\
Female & 0 \\
Unemployed & $1.50(1.09,1.92)^{* *}$ \\
Government employed & 0 \\
Unable to read and write & $0.21(-0.09,0.51)$ \\
College graduate and above & 0 \\
Age at first onset of illness & $-0.15(-0.20,-0.11)^{* *}$ \\
Duration of treatments & $-0.06(-0.11,-0.01)^{*}$ \\
More than two episodes & $0.95(0.61,1.29)^{* *}$ \\
First episode & 0 \\
Previous hospitalization & $1.61(1.18,2.04)^{* *}$ \\
No previous hospitalization & 0 \\
Positive syndrome & $0.10(0.06,0.33)^{* *}$ \\
Negative syndrome & $0.50(0.10,0.20)^{* *}$ \\
Depressive symptoms & $-2.50(-3.03,-2.05)^{* *}$ \\
No depressive symptoms & 0 \\
Poor social support & $-0.05(-0.57,0.48)$ \\
Moderate social support & $-0.11(-0.62,0.40)$ \\
Strong social support & 0 \\
\hline $\begin{array}{l}\text { Note: } V I F_{\text {max }}=3.51, \text { constant }=11.00 \\
\text { summary: } R^{2}=88 \%, \text { adjusted } R^{2}=87.6 \%, \text { and } F(12,432)=263.14 \\
\text { with } p \text { value }<0.001 . *{ }^{* *}=\text { statistically significant }(p \text { value }<0.001) \text { and } \\
*^{*}=\text { statistically significant }(p \text { value }<0.05) .\end{array}$ \\
\hline
\end{tabular}

in Italy [28]. The possible reasons could be aberrant positive symptoms of schizophrenia (hallucination or other symptoms of psychosis) and prefrontal dysfunction which affect neural transmission in Central nervous system that could altered working memory.

According to this study; having more than two episodes and previous hospitalizations were positively associated with mean total insight score. The findings were consistent with different studies in Bulgaria [29], Taiwan [22] and New York [12], stated that more than two episodes and previous hospitalization were significantly associated with poor insight. The results could be justified as the patient with schizophrenia has biopsychosocial causality, the nature of the illness, poor adherence to medication and poor social support systems.

This study also showed that age at first onset of illness and duration of treatments was inversely associated with the mean total insight score. Supported by different studies conducted in Canada [30], Italy [28], London [31], and USA [32] which showed that the older age of first onset and the long-term duration of treatments predicted good insight. The possible explanation of improvement of insight with long term duration of treatments is the effect of medication and the patient spends more time in a medical setting and hearing psychiatric terminology and they may tend to pick up relevant words as the result of learning effect. There might be increasing social interaction and health information seeking behaviors among older age that would be likely to decreases mean insight score.

The positive and negative syndromes of schizophrenia were positively associated with the mean insight score in this 
study. The findings were consistent with the different studies in Ghana [25], Canada [33], London [31] and USA [34] That showed the positive and negative syndromes of schizophrenia predicting poor insight. The possible reasons could be that positive and negative syndromes of schizophrenia are a result of a deficit in frontal cortex and cortical midline region of the brain that could also be the reasons for insight processing $[35,36]$.

The current study revealed that presence of depressive symptoms was negatively associated with mean total insight score. The finding was in agreement with the different studies in Ghana [25], Canada [33], Italy [37] and New York [12] stated that patients with more insight have depressive symptoms. The reason could be justified as the patient with schizophrenia has been characterized by multiple domains of symptoms. Depressive symptoms are most commonly presented at residual phase after controlling active symptoms of the schizophrenia. The patients are likely to start to regain their awareness toward mental illness and consequence of mental illness; as a result, they could internalize the situation and develop depression. This result was also in further support of "insight paradox" (describes the presence of depressive symptoms among patients with schizophrenia who have a good level of insight).

\section{Conclusion}

This study revealed that the mean total insight score was moderate. Unemployed, having more than two episodes, previous hospitalization, positive syndrome, negative syndrome, and medication nonadherence were positively associated with the mean total insight score whereas age at onset of illness, duration of treatments and presence of depressive symptoms were inversely associated with the mean total insight score among patients with schizophrenia.

\section{Limitation of the Study}

The limitation of this study might be that some variables are affected by recall bias and that the scale to assess unawareness of mental disorder is not validated in Ethiopia.

\section{Abbreviations}

AMSH: Amanuel mental specialized hospital

PANSS: Positive and negative syndrome scale

SD: $\quad$ Standard deviation

SPSS: Statistical package of social science

SUMD: Scale to assess unawareness of mental disorder

WHO: World health organization.

\section{Data Availability}

The data sets used and/or analyzed during the current study are available from the corresponding authors on reasonable request.

\section{Ethical Approval}

Ethical clearance was obtained from the joint ethical review committee of the University of Gondar and AMSH. A formal letter of permission was obtained from AMSH. informed verbal consent was obtained from study participants before making an interview. Information on the study was explained to the participants including potential risks and benefits of the study. The respondents were informed that they have the right to refuse participation in the study at any time and refusing to participate will not affect them.

\section{Conflicts of Interest}

The authors declare that they have no conflicts of interest.

\section{Authors' Contributions}

MT involved in designing and coordinating over all progress of the study; DD, TF, MM and DA equally contributed in the design of the study performed linear regression model and critically suggested the manuscript. All authors read and approved the final manuscript.

\section{Funding}

Funding for this study was provided by a joint program from University of Gondar and AMSH.

\section{Acknowledgments}

Our heartfelt thanks are to AMSH and University of Gondar for the financial support and giving us the opportunity to undertake this study. We are also highly indebted to all AMSH staff members and data collectors for their support and cooperativeness, during the data collection time. Finally, our appreciation goes to study participants, who are voluntarily responding to the questionnaire.

\section{References}

[1] B. J. Sadok, V. A. Sadock, and P. Ruiz, Synopsis of Psychiatry Behavioral Sciences/Clinical Psychiatry, p. 455, Lippincott Williams and Wilkin, philadelphia, PA, USA, 2014.

[2] P. F. Buckley, D. A. Wirshing, P. Bhushan, J. M. Pierre, S. A. Resnick, and W. C. Wirshing, "Lack of insight in schizophrenia: impact on treatment adherence," CNS Drugs, vol. 21, no. 2, pp. 129-141, 2007.

[3] T. M. Lincoln, E. Lüllmann, and W. Rief, "Correlates and long-term consequences of poor insight in patients with schizophrenia. A systematic review," Schizophrenia Bulletin, vol. 33, no. 6, pp. 1324-1342, 2007.

[4] S. A. Yale and J. M. Gorman, "Awareness of illness in schizophrenia," Schizophrenia Bulletin, vol. 17, no. 1, pp. 113-132, 1991.

[5] A. Xavier, I Am Not Sick I Dont Need Help, pp. 16-127, Vida Press, New York, NY, USA, 10th edition, 2012. 
[6] S. Pini, L. D. Osso, X. F. Amador, C. Mastrocinque, M. Saettoni, and G. B. Cassano, "Awareness of illness in patients with bipolar I disorder with or without comorbid anxiety disorders," Australian \& New Zealand Journal of Psychiatry, vol. 37, no. 3, pp. 355-361, 2003.

[7] A. Jablensky, N. Sartorius, G. Ernberg et al., "Schizophrenia: manifestations, incidence and course in different cultures a World Health Organization ten-country," Psychological Medicine Monograph Supplement, vol. 20, pp. 1-97, 1992.

[8] X. F. Amador, M. Flaum, N. C. Andreasen et al., "Awareness of illness in schizophrenia and schizoaffective and mood disorders," Archives of General Psychiatry, vol. 51, no. 10, pp. 826-836, 1994.

[9] World Health Organization, Schizophrenia and Public Health. Nations For Mental Health, pp. 1-32, WHO, Geneva, 1998.

[10] J. A. Engh, Cognitive insight and clinical insight in schizophrenia, pp. 1-116, University of Oslo, Oslo, 2010.

[11] L. Bucky, M. Bodnar, A. Malla, R. Joober, and M. Lepage, "A 12-month outcome study of insight and symptom change in first-episode psychosis," Early Intervention in Psychiatry, vol. 4, no. 1, pp. 79-88, 2010.

[12] F. Amador, M. Flaum, D. Ph, H. Strauss, A. Yale, and J. M. Gorman, "Assessment of insight in psychosis," American Journal of Psychiatry, vol. 150, pp. 873-879, 1993.

[13] M. A. F. De Hert, V. Simon, D. Vidovic et al., "Evaluation of the association between insight and symptoms in a large sample of patients with schizophrenia," European Psychiatry, vol. 24, no. 8, pp. 507-512, 2009.

[14] B. J. Cuffel, J. Alford, E. P. Fischer, and R. R. Owen, "Awareness of illness in schizophrenia and outpatient treatment adherence," Journal of Nervous and Mental Disease, vol. 184, no. 11, pp. 653-659, 1996.

[15] P. Michel, K. Baumstarck, P. Auquier et al., "Psychometric properties of the abbreviated version of the scale to assess unawareness in mental disorder in schizophrenia," $B M C$ Psychiatry, vol. 13, no. 1, 2013.

[16] S. R. Kay, L. A. Opler, and J. P. Lindenmayer, "Reliability and validity of the positive and negative syndrome scale for schizophrenics," Psychiatry Research, vol. 23, no. 1, pp. 99-110, 1988.

[17] A. Fekadu, M. Mesfin, G. Medhin et al., "Adjuvant therapy with minocycline for schizophrenia (The MINOS Trial): study protocol for a double-blind randomized placebo-controlled trial," Trials, vol. 14, no. 1, p. 406, 2013.

[18] D. Addington, J. Addington, and M. Atkinson, "A psychometric comparison of the calgary depression scale for schizophrenia and the hamilton depression rating scale," Schizophrenia Research, vol. 19, no. 2-3, pp. 205-212, 1996.

[19] D. Levine, L. Green, and D. Morisky, "Concurrent and predictive validity of a self-reported measure of medication adherence," Medical Care, vol. 24, no. 1, pp. 67-74, 1986.

[20] O. S. Dalgard, Explanation of oslo social support scale (OSS- 3): European Public Health Information System, pp. 1-6, 2008.

[21] J. Soriano-barceló and A. S. David, "Insight assessment in psychosis and psychopathological correlates: validation of the Spanish version of the schedule for assessment of insight expanded version," The European Journal of Psychiatry, vol. 30, no. 1, pp. 55-65, 2014.

[22] C. F. Yen, M. L. Yeh, C. S. Chen, and H. H. Chung, "Predictive value of insight for suicide, violence, hospitalization, and social adjustment for outpatients with schizophrenia: a prospective study," Comprehensive Psychiatry, vol. 43, no. 6, pp. 443-447, 2002.

[23] A. W. B. Van Baars, A. I. Wierdsma, M. W. Hengeveld, and C. L. Mulder, "Improved insight affects social outcomes in involuntarily committed psychotic patients: a longitudinal study in the Netherlands," Comprehensive Psychiatry, vol. 54, no. 7, pp. 873-879, 2013.

[24] N. Crumlish, P. Samalani, A. Sefasi, A. Kinsella, E. O. O'Callaghan, and H. Chilale, "Insight psychopathology and global functioning in schizophrenia in urban Malawi," British Journal of Psychiatry, vol. 191, no. 3, pp. 262-263, 2007.

[25] N. A. Poole, J. Crabb, A. Osei, P. Hughes, and D. Young, "Insight, psychosis, and depression in Africa: a cross-sectional survey from an inpatient unit in Ghana," Transcult Psychiatry, vol. 50, no. 3, pp. 433-441, 2013.

[26] Y. T. Xiang, Y. Wang, C. Y. Wang et al., "Association of insight with sociodemographic and clinical factors, quality of life, and cognition in Chinese patients with schizophrenia," Comprehensive Psychiatry, vol. 53, no. 2, pp. 140-144, 2012.

[27] A. O. Adewuya, B. A. Ola, S. K. Mosaku, F. O. Fatoye, and A. B. Eegunranti, "Attitude towards antipsychotics among out-patients with schizophrenia in Nigeria," Acta Psychiatrica Scandinavica, vol. 113, no. 3, pp. 207-211, 2006.

[28] S. Pini, G. B. Cassano, L. Dell'Osso, and X. F. Amador, "Insight into illness in schizophrenia, schizoaffective disorder, and mood disorders with psychotic features," American Journal of Psychiatry, vol. 158, no. 1, pp. 122-125, 2001.

[29] I. V. Vassileva and V. K. Milanova, "Attitudes toward antipsychotic medication, insight and psychopathology in outpatients with schizophrenia," Folia Medica, vol. 54, pp. 62-68, 2012.

[30] P. Gerretsen, E. Plitman, T. K. Rajji, and A. Graff-guerrero, "the effects of aging on insight into illness in schizophrenia: a review," International Journal of Geriatric Psychiatry, vol. 29, no. 11, pp. 1145-1161, 2014.

[31] M. Sanz, R. Kemp, and A. David, "A comparative study of insight scales and their relationship to psychopathological and clinical variables," Psychological Medicine, vol. 28, no. 2, pp. 437-446, 1998.

[32] J. P. McEvoy, J. Johnson, D. Perkins et al., "Insight in firstepisode psychosis," Psychological Medicine, vol. 36, no. 10, pp. 1385-1393, 2006.

[33] A. R. Mintz, K. S. Dobson, and D. M. Romney, "Insight in schizophrenia: a meta-analysis," Schizophrenia Research, vol. 61, no. 1, pp. 75-88, 2003.

[34] A. Garcia and P. H. Lysaker, "Socially naïve self-appraisal moderates the relationship between cognitive insight and positive symptoms in schizophrenia," Schizophrenia Research, vol. 143, no. 1, pp. 97-101, 2013.

[35] K. Osatuke, J. Ciesla, J. W. Kasckow, S. Zisook, and S. Mohamed, "Insight in schizophrenia: a review of etiological models and supporting research," Comprehensive Psychiatry, vol. 49, no. 1, pp. 70-77, 2008.

[36] A. S. David, L. Van Der Meer, and S. Costafreda, "Self-reflection and the brain: a theoretical review and meta-analysis of neuroimaging studies with implications for schizophrenia," Neuroscience \& Biobehavioral Reviews, vol. 34, no. 6, pp. 935-946, 2010.

[37] M. Belvederi Murri, M. Respino, M. Innamorati et al., "Is good insight associated with depression among patients with schizophrenia? Systematic review and meta-analysis," Schizophrenia Research, vol. 162, no. 1-3, pp. 234-247, 2015. 


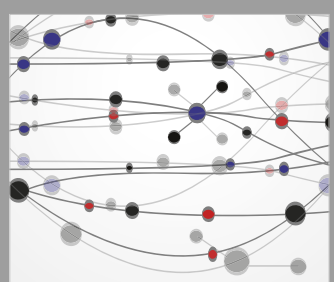

The Scientific World Journal
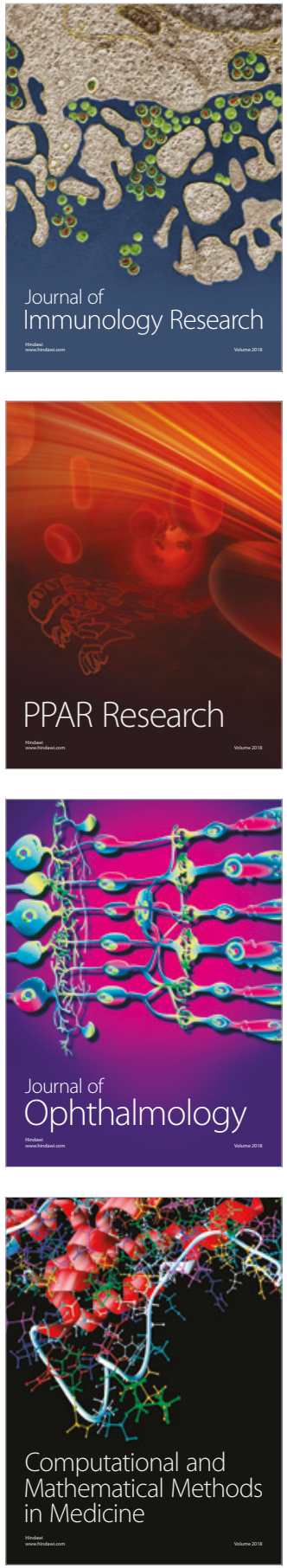

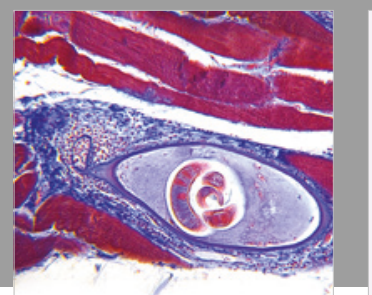

Gastroenterology Research and Practice

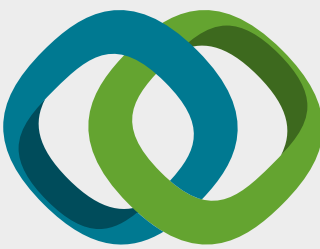

\section{Hindawi}

Submit your manuscripts at

www.hindawi.com
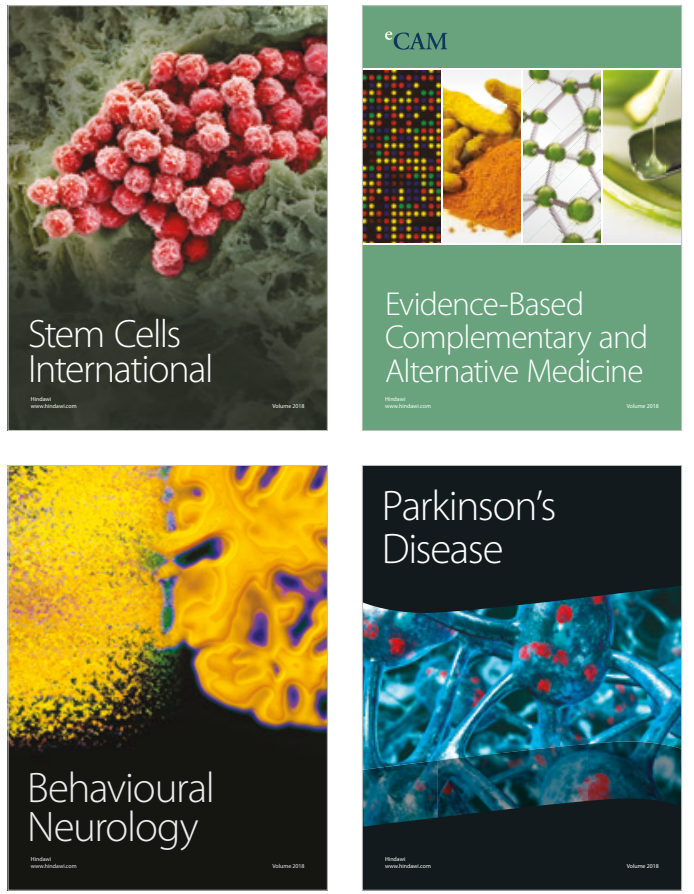

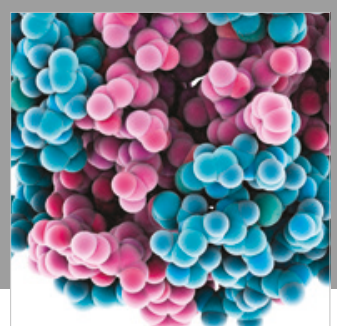

ournal of

Diabetes Research

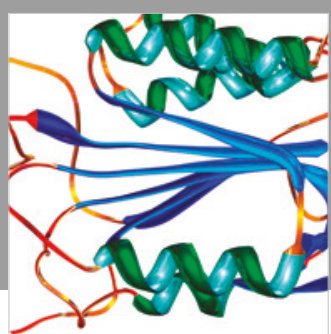

Disease Markers
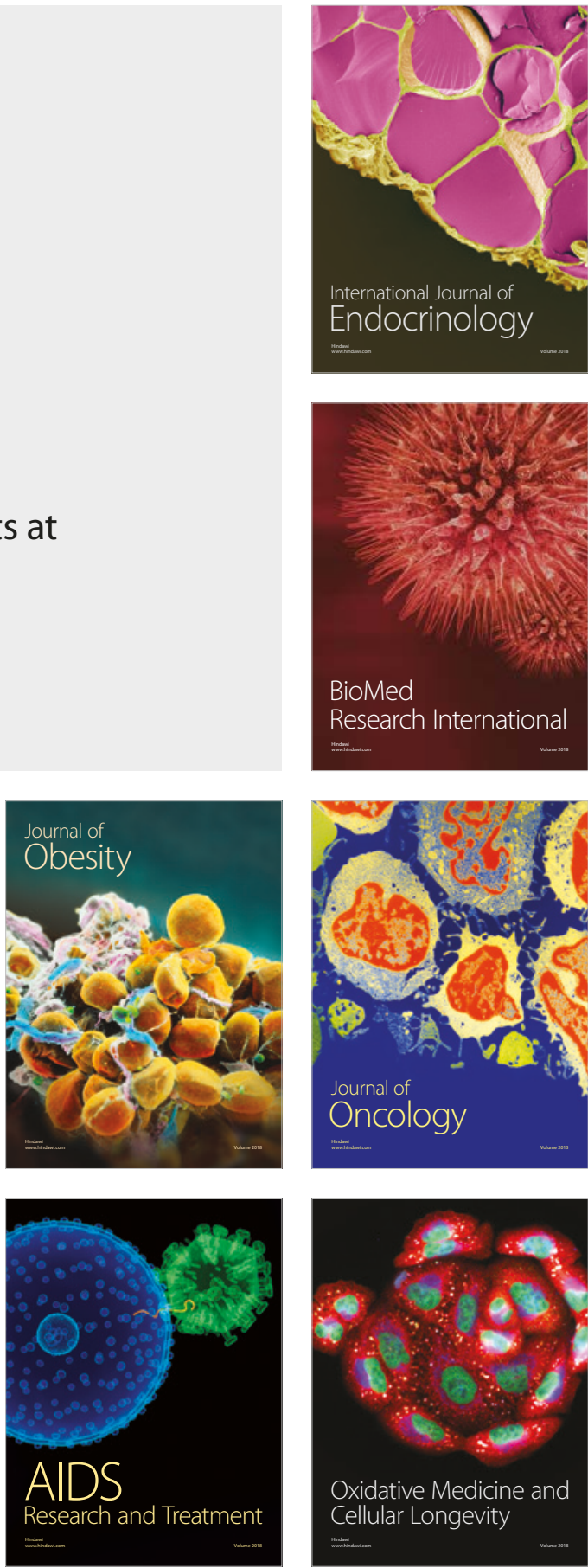